\title{
Association between organophosphate exposure and hyperactivity?
}

\section{To the editor:}

Winrow and coworkers ${ }^{1}$ have offered a potentially useful genetically modified mouse model for study of the health implications of altered expression of neuropathy target esterase (Nte). But their primary conclusion that "moderate reduction in Nte activity, by either reducing the amount of Nte protein through genetics or using a potent Nte inhibitor, leads to hyperactivity" is critically flawed. The key data justifying their conclusion, presented in Figure $6 \mathrm{c}$ and $\mathrm{d}$, showed that wild-type $\left(\mathrm{Nte}^{+/+}\right)$mice treated intraperitoneally with $1 \mathrm{mg}$ ethyl octylphosphonofluoridate (EOPF) per kg body weight had a hyperactivity response equal to or greater than that observed in untreated genetically engineered $\mathrm{Nte}^{+/-}$mice with $40 \%$ lower intrinsic Nte enzymatic activity. Although it was quantified in untreated $\mathrm{Nte}^{+/-}$mice, Nte activity was not reported in the EOPFtreated mice. Evidence for Nte inhibition in EOPF-treated mice was only inferred by reference to results of an earlier study ${ }^{2}$ in which intraperitoneal treatment with $5 \mathrm{mg}$ of EOPF per $\mathrm{kg}$ body weight was described as inhibiting NTE activity in mouse brain by $85 \%$. But in the same table that describes this response (Table 4 in ref. 2),

intraperitoneal treatment with $1.3 \mathrm{mg}$ EOPF per kg body weight is reported as causing no inhibition of Nte activity in mouse brain (only 1\% difference from control). This dose of $1.3 \mathrm{mg}$ per $\mathrm{kg}$ body weight is slightly higher than that used in the hyperactivity experiments described by Winrow et al. ${ }^{1}$.

These data on EOPF and Nte inhibition suggest that activity of Nte in the brain was probably not reduced at the dose used in the experiments ascribing increased hyperactivity to Nte inhibition induced by EOPF treatment. In the absence of measurements of inhibition of Nte activity in the brain at a dose of $1 \mathrm{mg}$ EOPF per kg body weight, and knowing that a dose of $1.3 \mathrm{mg}$ EOPF per $\mathrm{kg}$ body weight did not inhibit activity of Nte in the brain in other similar experiments, the hypothesis that organophosphate-induced inhibition of Nte is causally linked to hyperactivity is not plausible.

James Bus ${ }^{1}$, Jacques Maurissen
Brian Marable $^{1}$ \& Joel Mattsson

${ }^{1}$ The Dow Chemical Company, Midland, Michigan 48674, USA. ${ }^{2}$ Dow AgroSciences, Indianapolis, Indiana 46268, USA.

1. Winrow, C.J. et al. Nat. Genet. 33, 477-485 (2003).

2. Wu, S.-Y. \& Casida, J. Toxicol. Appl. Pharmacol. 139, 195-202 (1996).

\section{In reply}

We appreciate the interest and enthusiasm resulting from our report describing the generation of Nte-haploinsufficient $\left(\mathrm{Nte}^{+/-}\right)$ mice. In their letter, Bus et al. conclude that "the hypothesis that organophosphateinduced inhibition of Nte is causally related to hyperactivity is not plausible". We disagree on the basis of four lines of evidence. First, they did not note that the earlier study used Swiss-Webster mice and the present study used 129S6/SvEvTac mice. We know that the activity of Nte in the brain is different in these two strains, and differences in detoxifying enzymes might also contribute to any apparent dose-response discrepancy.
Second, we show that $\mathrm{Nte}^{+/-}$mice have elevated motor activity relative to $\mathrm{Nte}+/+$ littermates. These mice are genetically identical except for Nte haploinsufficiency. The power of mouse genetics enables analysis of the effects resulting from manipulating a single gene through comparisons with a littermate that is otherwise genetically identical. As noted in their letter, the Nte ${ }^{+/-}$ mice have a $40 \%$ reduction in Nte activity in brain homogenates. From these results, it can be concluded that a decrease in Nte levels and activity leads to an increase in motor activity. Third, when $\mathrm{Nt} e^{+/+}$mice are exposed to $1 \mathrm{mg}$ of EOPF per kg body weight, we again see an increase in motor activity similar to that seen with $\mathrm{Nt} e^{+/-}$mice. EOPF has been shown to be a potent Nte inhibitor both in vitro and in vivo in Swiss-Webster mice and hens. Finally, $\mathrm{Nte}^{+/-}$mice are more sensitive than their $\mathrm{Nte}^{+/+}$littermates to the toxic effects of EOPF at $6-10 \mathrm{mg}$ per $\mathrm{kg}$ body weight. The overlap between the genetic and EOPF exposure experiments leads directly to the hypothesis that inhibition of Nte, either chemically or genetically, can lead to hyperactivity.

\author{
Christopher J Winrow ${ }^{1,3}$, John E Casida ${ }^{2}$ \\ \& Carrolee Barlow ${ }^{1,3}$
}

${ }^{1}$ The Salk Institute for Biological Studies, The Laboratory of Genetics, 10010 North Torrey Pines Road, La Jolla, California 92037, USA.

${ }^{2}$ Environmental Chemistry and Toxicology Laboratory, Department of Environmental Science, Policy and Management, 115 Wellman Hall, University of California, Berkeley, California 94720-3112, USA. ${ }^{3}$ Present address: Merck Research Laboratories, 3535 General Atomics Court, San Diego, California 92121, USA. 